\title{
Clinical Usefulness of Fecal Microbiota Transplantation
}

\author{
Jung Ho Park \\ Department of Internal Medicine, Kangbuk Samsung Hospital, Sungkyunkwan University School of Medicine, Seoul, Korea
}
Article: Serial frozen fecal microbiota transplantation in the treatment of chronic intestinal pseudo-obstruction: a preliminary study
Gu L, Ding C, Tian H, et al
(J Neurogastroenterol Motil 2017;23:289-297)

Fecal microbiota transplantation (FMT) is the transfer of stool from healthy donors to patients with an altered colonic microbiome to cure a specific disease. The main goal of FMT is to restore the "normal" population of bacteria in a dysbiotic colonic environment. Until now, the strongest evidence for efficacy of FMT is the treatment of Clostridium difficile infection (CDI), especially for recurrent ones. ${ }^{1}$ The results showed that FMT was successful in $85 \%$ of recurrent CDI and $55 \%$ of refractory CDI compared with $30 \%$ to $80 \%$ success rates for medical therapies. ${ }^{2}$ Other possible applications for FMT may include diseases associated with dysbiosis such as inflammatory bowel disease, irritable bowel syndrome, Parkinson's disease, and metabolic syndrome.

In the current issue of Journal of Neurogastroenterology and Motility, Gu et $\mathrm{al}^{4}$ hypothesized that manipulating the gut microbiome through FMT could reverse intestinal dysbiosis and consequently resolve overt chronic intestinal pseudo-obstruction (CIPO). Nine patients with CIPO received FMT for 6 consecutive days through nasojejunal tubes and were followed up for 8 weeks after treatment. FMT significantly alleviated pain and bloating symptoms, but not vomiting and nausea. Enteral nutrition administered through a nasojejunal tube after FMT was well-tolerated by $66.7 \%$ (6/9) of patients. Computed tomography scores of intestinal obstructions were significantly reduced after FMT and small intestinal bacterial overgrowth (SIBO) was eliminated in $71 \%(5 / 7)$ of patients.

Based on these findings, the author suggested that serial FMT may be a candidate therapy for CIPO. However, there are a few things to consider. We cannot agree with the hypothesis that if intestinal dysbiosis is reversed, then CIPO might be resolved. As mentioned in the introduction, the etiology of symptoms in CIPO is multifactorial, and dysbiosis is the result of CIPO, not the cause. Also, this study had several limitations, as the author said: small number of cases, no control group, short follow up period, and more than anything else, heterogeneity of participants. Of the 9 patients who participated in this study, 6 underwent surgical treatment, including ileostomy in 4 patients. These surgical procedures could have a great effect on the colonic bacteria.

Nonetheless, it is interesting to note that resolving SIBO with FMT could improve the patient's nutritional status of CIPO and FMT may be a good treatment option for patients with symptoms

Received: February 20, 2107 Revised: None Accepted: February 23, 2017

↔ This is an Open Access article distributed under the terms of the Creative Commons Attribution Non-Commercial License (http://creativecommons. org/licenses/by-nc/4.0) which permits unrestricted non-commercial use, distribution, and reproduction in any medium, provided the original work is properly cited.

${ }^{*}$ Correspondence: Jung Ho Park, MD Department of Internal Medicine, Sungkyunkwan University School of Medicine, Kangbuk Samsung Hospital, 29 Saemunan-ro, Jongno-gu, Seoul 03181, Korea

Tel: +82-2-2001-2059, Fax: +82-2-2001-2485, E-mail: pjho3@hotmail.com 
that are associated with SIBO.

\section{Financial support: None.}

\section{Conflicts of interest: None.}

\section{References}

1. Gough E, Shaikh H, Manges AR. Systematic review of intestinal mi- crobiota transplantation (fecal bacteriotherapy) for recurrent Clostridium difficile infection. Clin Infect Dis 2011;53:994-1002.

2. Drekonja D, Reich J, Gezahegn S, et al. Fecal microbiota transplantation for Clostridium difficile infection: a systematic review. Ann Intern Med 2015;162:630-638.

3. Vindigni SM, Surawicz CM. Fecal microbiota transplantation. Gastroenterol Clin North Am 2017;46:171-185.

4. Gu L, Ding C, Tian H, et al. Serial frozen fecal microbiota transplantation in the treatment of chronic intestinal pseudo-obstruction: a preliminary study. J Neurogastroenterol Motil 2017;23:289-297. 